\title{
Sobre l'autoría de Píramu y Tisbe
}

\author{
por Xuan Xosé Sánchez Vicente
}

$\mathrm{V}$

AMOS eNTAMAR pelo que ye pa mi lo más evidente y, a la vegada, lo más cenciello argumentalmente: cuando lleíl Sueños de Nabucodonosor, de Benito de L'Auxa, y lu comparé con Píramu y Tisbe na edición de Xuan Carlos Bustor, lo que camenté inmediatamente ye que l'autor de Sueños de Nabucodonosor nun podía ser el mesmu de la composición heredada de les Metamorfosis d'Ovidiu.

Peles mesmes dómines, Antón García, nuna reseña a la edición de Xuan Carlos Busto 2 , terminaba diciendo:

«Na cuestión de l'autoría d'esi poema nun resulta fácil tomar partíu, porque si bien les daes qu'apurren Viejo y Busto parecen dar la razón a la so tesis, la llectura de los textos, anque tengan l'aire familiar d'una mesma época y de similares llectures e influencies, fainos albidrar mayor pericia y aciertu nel manexu del versu quienquiera que fuera l'autor de "Píramo y Tisbe", frente al estilu daqué frayosu del "Nabucodonosor"”.

Y más de recién, nel 20I3, Pablo Suárez García, nun trabayu d’indagación estadística sobre autoríes, «La identificación d’autoría

' Benito de L’Auxa, Antón Balvidares, Poesies. Edición de Xuan Carlos Busto, Gobiernu del Principáu d'Asturies y Ediciones Trabe, Uviéu, 2012.

2 Antón García, «Dos poetes de la Ilustración asturiana», en La Nueva España del o4/o6/ıor2. 
n'asturianu» ${ }^{3}$, daba una probabilidá del 95,037 de que l'autoría del Píramи y Tisbe fuere de Marirreguera. Masque hai que señalar que l'estudiu taba fechu enantes del asoleyamientu en 2012 por Xuan Carlos Busto del Sueños de Nabudonosor y, polo tanto, el poema d'Auxa nun entrara nel conxuntu de datos colos que la máquina ficiera les comparances y estableciera les probabilidaes d'atribución. Con too y con ello, el datu d'una identificación tan alta de Marirreguera como probable autor de Píramu y Tisbe ye, de por sí, un argumentu de pesu.

\section{La escritura de Sueños de Nabucodonosor}

Pero vamos desaminar dalgunos de los aspectos del Sueños... que nos paecen d'una mano poco curiosa, o, si se quier, d'eso qu'Antón García calificaba d' «estilu frayosu».

Vamos entamar pol usu y «abusu» la mitoloxía.

\section{Mitoloxía}

Atopamos nel testu un total de ventiún referencies a la mitoloxía grecollatina: diecinueve a personaxes ${ }^{4}$, dos a llugares; trés d'elles, dos de personaxes, otra de llugares, fáense al través de perífrasis.

Pero non solo ocurre esa bayura de cites mitolóxiques nel conxuntu’l testu. A vegaes atrópense en pocos versos, lo que paez un usu ensin

3 Pablo Suárez García, «La identificación de l'autoría n’asturianu», Lletres Asturianes, n. ${ }^{\circ}$ Io9, Uviéu (Academia de la Llingua Asturiana), 2013.

${ }_{4}$ Ye duldosa la calificación que puede tener el «sol» de la estrofa 48, pues si bien ye aparentemente l'astru («Mirá el sol, qué carreres va tirando... lluigu irá a otres antípodes llumando / ñon tardará en tapase con lles cuentes», vss. 379, 38I, 382), per otru llau ye’l Sol mitolóxicu: «Mirá el sol, qué carreres va tirando / a decir a so Cronis cuatro fiestes», esto ye, Apolo, el dios del sol, del que Cronis ye la so infiel amante. 
munchu tinu: nada menos que cuatro referencies (trés nomes propios y una perífrasis) s'amontonen nestos trés versos':

«[podía] Pasar de baldre la negra llaguna, / como al son de la llira pasó Orfeo / y, polla fuerza, Alcides y Teseo» (vss. I4 a I6).

Estes son les restantes ocasiones; dalguna d'elles requier un ciertu nivel de conocencia pa tener les claves d'a quién se refier o pa saber la historia de la que formen parte.

«Ñon vigo aquella diosa dormiolenta, / que con teyes ardiendo y gran fatiga / buscó a una fiya suya, tal añada, / por más tierra qu'en mundo ves sembrada» (vss. 2I a 24).

«[Nabucodonosor] sobremanera era soñolinto / con lles hisopiadures de Morfeo» (vss. 69 a 70).

«¿Xamás pasaste pel Llano del Monte / cuando cuecen el barro ña teyera? / y al llado, da u'l gorgoxo cai lla fonte, / verís abierto a modo de montera / un grandísimo pozo, que Aqueronte, / anque afinque so barca ña ribera / del Leteo y a Plutón mire lla cama, / ñon vería desbuchar tan grande llama» (vss. I85 a 192).

«Folgábase Nabuco a so placencia, / como solía Vertumno con Pomona» (vss. 227 y 228).

«Ñon era menester allí el cuidado / de Bato, ñin la llinde falta fía / d'aquel otru pastor, que descuidado / dio llugar de facer so fechuría / a Mercurio, lladrón descomulgado / que concencia ñin alma ñon tenía, / ñon dexando ñi aún delles verraques / del rei Admeto, y otros furtar vaques» (vss. 257 a 264).

«Mas muéva-yos tamién el desperdicio / de la lloramicona, que del viento / diz que ye madre, y de Titón fenoya, / fío de Laodemonte, rei de Troya» (vss. 293 a 296).

Lo sorprendente d'estes dos caberes cites consiste en que ye'l propiu rei Nabucodonosor el que les emplega al narrar el so sueñu. Como se

s Por cierto, y como dixéramos yá nel so día, esta presencia la mitoloxía fai simplemente risible la idea de qu'estos poemes s'emplegaren pol cleru p'adoctrinar a los parroquianos, al pueblu ordinariu. 
ve, el decoru o verosimilitú de la escritura padez abondo con esti tan desapropiáu usu.

A ello podíemos arreya-y les referencies cultes pseudohistóriques qu'equí vemos nes dos siguientes cites, feches, nin más nin menos, que pol xefe d'una cuadriella trabayadores, que ye, precisamente, el narrador del conxuntu'l relatu:

«Aquisti año en qu'estamos se m'antoxa / a uno d'aquellos de la Edá Tercera / en que ñon se dexó macón ñin goxa, / entresuilu, desván, horro o panera / por llenar...» (vss. 25 a 29).

"QQué bien vos afayáis non trabayando!, / deben plizcabos muncho lles arestes. / Mirá el sol, qué carreres va tirando / a decir a so Cronis cuatro fiestes» (vss. 377 a 380).

L’usu de la parénesis a los personaxes/oyentes, común nos testos clasicistes de Marirreguera y en Píramu y Tisbe, el que ta en discusión, ye equí, acasu, desaxeráu.

\section{Parénesis a los personaxes/oyentes}

La narración entama col marcu endoculturador marirreguerianu. Y ehí faense ocho apelaciones a los oyentes del narrador, cuatro xenerales (en vós) y otres cuatro particulares (a Xuan, Estebano, Llocía, Antonia).

«Nun veréis, anque más abráis el güeyo, / mozo que faga tal ñisti conceyo» (vss. 7 a 8 ).

«Quita la mesa Xuan!, ¡qué bon paxello!» (v. 37).

«iEstebano!, en tanto que s'aposienta / lla comida que Dios quixo güe daños, / vente so isti ñozal, que'l sol calienta / y a fee que da ganancia a ciruxanos. / Tu, Llocía, tamién estáme atenta, / vosotros dexá yá el xugar de manos, / cada cual ponga a un llado so mesoria / y oí, que vo cuntavos una hestoria» (vss. 4I a 48).

«Mas antes d'empezar sabé primero» (v. 57).

«Hora bien, atendé, ¡cuenta me llamo!, / nadie me diga chit, ¡cierra Candamo!» (vss. 63 a 64 ).

«(non pigaces, Antona, bien te veo)» (v. 66). 
«Y si me preguntáis de qué tenía / los pies, vos lo diré, que non lo ñoro» (vss. I25 y I26).

“¿Xamás pasaste pel Llano del Monte / cuando cuecen el barro ña teyera? / y al llado, da u'l gorgoxo cai lla fonte, / verís abierto a modo de montera / un grandísimo pozo, que Aqueronte, / anque afinque so barca ña ribera / del Leteo y a Plutón mire lla cama, / ñon vería desbuchar tan grande llama» (vss. I85 a I92).

Y piesllen la narración trés momentos de nueves apelaciones a los personaxes/oyentes; d'ente ellos hai ún nel que se llama a un nuevu personaxe, Mingón:

«A nosotros el tiempo nos apierta / qu'hemos dir güe dormir a lla Machina. / ¡Llevantá!, ffe pumar!, ;mesoria al brazo! / y vamos a coyer aquel pedazo» (vss. 373 a 376).

"¿Qué bien vos afayáis non trabayando!, / deben plizcabos muncho lles arestes. / Mirá el sol, qué carreres va tirando / a decir a so Cronis cuatro fiestes; / lluigu irá a otros antípodes llumando, / ñon tardará en tapase con lles cuestes. / ¡Ea!, agarre cadún el so sombriro, / que no ha quedar güe espiga a isti suquiro» (vss. 377 a 384).

«Atira acá, Mingón, lla tabaquera, / a ver si se me escuerre esta pereza, / que’l cuntar cuentos llargos a cualquiera / cría farria y pesariellos de cabeza; / mas, con todo eso, el xueves eña era / tengo decevos d'otra bona pieza, / qu'estando xecutando una ensolencia / vigo ña paré escrita so sentencia» (vss. 385 a 392).

\section{Intervenciones del narrador (primer persona)}

Les intervenciones del narrador en primer persona comentando la historia qu'él mesmu cuenta o enxertando la so propia esperiencia pa esclariar lo narrao son, asinamesmo, acaso reiteraes n'escesu y, dende'l mio puntu de vista, munches d'elles inadecuaes.

«Llarga de cuirpu a modo de xigante / lles costielles y brazos (cosa rara) / de plata fina: ñon gastaba guantes / porque yera manquina, pero aquesto / bon misterio tendría, yo lo apuesto» (vss. 116 a I20).

«Y si me preguntáis de qué tenía / los pies, vos lo diré, que non lo ñoro» (vss. I25 y I26).

«Dio-y de Babilonia el mando tudo, / ficho yera a gobernalla, ñon lo dudo» (v. I44). 
«Nunca caliro enciso aquí ña Braña / por más que par atrás lla vista torno / cuando vo par Uviedo desde'l Viso, / anqu'estén arroxándolo, deviso» (vss. I8I a I84).

“¿Xamás pasaste pel Llano del Monte / cuando cuecen el barro ña teyera? / y al llado, da u'l gorgoxo cai lla fonte, / verís abierto a modo de montera / un grandísimo pozo, que Aqueronte, / anque afinque so barca ña ribera / del Leteo y a Plutón mire lla cama, / ñon vería desbuchar tan grande llama» (vss. I85 a 192).

«Pero, ¡o! ¡Qué gran milagro y maravía!, / maldita la migaya se quemano» (vss. 197 y 198).

«Pasiaben el camín, qu'eso diz qu'era, / como dicen, cual Pedro per so casa» (vss. 2OI a 202).

Mui singular ye ensin dulda esta apelación al llector del mesmu autor, al final del testu, y yá fuera de lo que ye propiamente'l poema, a la que sigue una declaración de sumisión al criteriu la Ilesia:

«Y tu, amado lector, que este poema / leído has, sus errores dilatados / disimula benigno, afable, honroso, / generoso y prudente, por lo Llanos» (vss. 393 a 396).

«Omnia S. Romane E. et prudentium judicio subficio».

Dalgunos testos de Reguera (polo menos talo como los conservamos nos manuscritos, non na edición de Caveda, que los esanició) tienen al final una conclusión o epitafiu ${ }^{6}$ empobináu al llector, onde se resume la «moralexa» del testu. Lo peculiar d'esti epitafiu de Benito de L'Auxa son dos coses: el que tea en castellanu y el que nun sea un resume o moralexa del relatu, sinón una captatio benevolentiae a posteriori ( $¡$ acaso a una persona concreta? ¿Xovellanos?).

${ }^{6}$ Tamién La Xudí de Xuan González Villar y Fuertes concluye con una décima que tien por títulu «epitafiu». Ver Xuan González Villar y Fuertes, La Xudit (1770), edición d’Antón García, Xixón (Llibros del Pexe), 1990. Por cierto, l'epitafiu que s'asoleya nesta edición nun provién del manuscritu col que trabaya Antón García, onde nun ta, sinón del Bosquejo... de Máximo Fuertes Acevedo, que lu trescribe ellí d'un manuscritu qu'él tenía, manuscritu del que desconocemos güei'l paraderu. 


\section{Procedimientos d'endoculturación}

Los procedimientos d'endoculturación son bayura. El más notable ye, como en Marirreguera, l'estructural, l'allugamientu del (semi)relatu bíblicu nun marcu cultural asturianu, nesti casu nuna tropiella de segadores qu'escuchen el cuentu del cabezaleru la cuadriella. Tamién como en Marirreguera ${ }^{7}$, el marcu endoculturador abre y cierra'l testu.

Pero amás d'esti procedimientu estructural atópense otros munchos d'estremada mena. El símil parabólicu marirreguerianu o símil de raigañu homéricu-virxilianu con apelación a los oyentes (vss. I85 a I93), o'l símil parabólicu implícitu (onde nun hai conclusión espresada) (vss. I8I a I84).

«¿Xamás pasaste pel Llano del Monte / cuando cuecen el barro ña teyera? / y al llado, da u'l gorgoxo cai lla fonte, / verís abierto a modo de montera / un grandísimo pozo, que Aqueronte, / anque afinque so barca ña ribera / del Leteo y a Plutón mire lla cama, / ñon vería desbuchar tan grande llama» (vss. 185 a 192).

«Nunca caliro enciso aquí ña Braña / por más que par atrás lla vista torno / cuando vo par Uviedo desde'l Viso, / anqu'estén arroxándolo, deviso» (vss. I8I a I84).

Pero lo más sorprendente son les intervenciones de Nabucodonosor falando como un contemporaneu. Asina, nes estrofes 3I a 39, onde, aparte otres "gracies», como tratar de "amigo» a Daniel, emplega la mitoloxía grecollatina, según yá se señalara, o la visión antigua y fabuladora del camaleón como ser que vive del vientu. $\mathrm{O}$, por apuntar lo más bultable, diz que l'árbol que viera nel so suañu fai pequeñu como una "faba» al carbayu mayor (v. 242), o qu'embaxu esi arbolón podía asolombrase «cualquiera romería» (v. 248), o que sintiera un «santo dando voces, que podía / oíse desde Uviedo hasta Llugones» (vss. 283 y 284).

7 Tanto en Dido y Enees, como en Hero y Lleandro y, de ser suyu, como nos paez, Píramu y Tisbe. 
La siguiente baragaña desamina lo que, al xuiciu del autor d'esti estudiu, son imprecisiones na espresión, de mou y manera que nun se sabe bien lo que quier dicir el testu, bien por escuru, bien por ambiguu

\section{Imprecisiones}

«PParñós!, ¡corrego bien Medero el canto!, / toda la andecha se quedó atordida, / coles mueques que fixo, ñon m'espanto / que la tuviés una hora devertida» (vss. I a 4 ).

¿Quedó l'andecha «atordida» col cantu o coles mueques? ¿Qué ye, facía mueques mientres cantaba? ¿O hai qu'interpretar que son les mueques, dempués del canciu, lo que tien a l'andecha «una hora devertida»?

«El miércores Pachón ñon bailó tanto, / porque isti güe dio saltos sin medida; / non veréis, anque más abráis el güeyo, / mozo que faga tal nisti conceyo» (vss. 5 a 8).

¿Quién ye «isti güe»? ¿Ye Pachón? ¿Ye otru? ¿Si ye otru -«isti»- quién ye? Si ye Pachón, ¿ye que baillar ye "dar saltos sin medida»? Pero si ye Pachón, ¿por qué «ñon bailló tanto»? ¿Ye la causa «porque dio saltos sin medida»?

«QQuita la mesa Xuan!, ¡qué bon paxello!» (v. 37).

¿A qué se refier el «qué bon paxello»? ¿Pue ser el significáu lliterariu tradicional de 'traxe de fiesta' o'l más corriente de 'prenda de vistir'? Nun tien contestu pa ello. ¿Refierse al mantel de la mesa? Inorámoslo. ¿Pero tien mantel na mesa un grupu de xornaleros qu'anden andechando?

${ }^{8}$ La interpretación qu'a "paxellu» da Xuan Busto na so edición del testu nun paez ser l'afayadiza. Anota: «'trapu vieyu, rotu' (DLLA, s. v., $4 \cdot^{\text {a }}$ ac.)». ¿Sería entós irónicu’l «qué bon paxello»? Pero, con too, siguimos entrugándonos cuála ye la verosimilitú d'un mantel na xinta d'esa tropiella. D'otru llau, les acepciones qu' escueye pa "paxellu» l'Academia (mayoritariamente col sentíu de 'trapu de pocu valor') nun concuayen coles que son les acepciones mayoritaries de la palabra (tanto na llingua oral como lliteraria) que puen vese nel DGLLA, que tienen, de forma 
¿Significa ehí paxello 'trapu vieyu o rotu', que ye la cuarta acepción de la palabra nel DLLA, como interpreta Xuan Busto, l'editor del poema? Y si ye asina, ¿qué sentíu tien el «qué bon paxello!»? ¿Ye l'oxímoron una ironía?

N'otros casos la imprecisión nun ta na ambigüedá o escuridá del testu, sinón na forma poco afayadiza de denomar una realidá, lo inapropiao de los términos con que se diz una cosa que sí ta clara. Asina:

[Nabucodonosor mira pal fornu onde tán los tres mozos condenaos a morrer] «mas cuando maxinaba que la llana / del vestido quemada yá estaría, / ablucóse y plasmóse al ver que, en seña / del fuibo, ñon tenín nin una angüeña» [estrofa 27, vss. 213 a 216$]$.

Vamos dexar a un llau esa inverosimilitú del rei mirando dende la ventana del so palaciu y viendo l'interior del fornu («que llocía seis llegües en contornu»). Pero, con esi fueu y el tiempu que llevaben dientro, dicir que pensaba que tendríen «quemada la llana del vestido» ye una imprecisión, un desplicase de manera tan aproximatible, que resulta poco aceptable.

Salíos yá Azaríes, Ananíes y Misael del fornu y acabada la narración d'esi episodiu y les sos consideraciones al respective, asina entama la estrofa 29:

«Desque s’asosegó esta pendencia / socedego otra tala, y ñon tan bona».

¡"Pendencia»? Bien poco apropiada ye la palabra pa designar el castigu de los xóvenes a ser amburaos nel fornu y la so salvación milagrosa.

Y de la palabra «camarada» del versu 34, na siguiente estrofa, la 29, podíamos dicir lo mesmo:

xeneral el sentíu de 'vistíu' (esternu) o 'prenda de vistir interior', a vegaes con sentíu positivu, 'prenda de calidá', 'prenda de vistir', que ye'l más corriente na lliteratura del xix y xx. 
«[El rei] Acodigo al remedio que solía / entrugando a Daniel, so camarada»"

El poema vien cerrase na estrofa 49, ensin acabar de parafrasiar el Llibru de Daniel, porque queda ensin contar, polo menos, l'episodiu acaso más conocíu de tolos del testu, la cena'l rei Baltasar, cuando ve apaecer una mano qu'escribe na paré unes frases que señalen el fin del so reináu, el famosu «mené, tequel, farsín», 'contáu, pesáu, dividíu' (na versión de la Biblia en castellanu que remano equí ${ }^{\mathrm{io}}$ ).

Pues bien, esa ausencia soluciónala con estos cuatro versos finales que vamos ver darréu, onde s'anuncia la parte central del episodiu, «vigo ña paré escrita so sentencia», pero onde'l momentu y el motivu del castigu que s'anuncia, que yera la profanación de los vasos sagraos que se llevaren a Babilonia xunto cola población xudía dempués de la destrucción del templu de Xerusalén pol padre de Baltasar, Nabucodonosor, ta representáu por esi imprecisu (pal llector o oyente que nun sepa yá la historia) «ensolencia».

«Mas, con todo eso, el xueves eña era / tengo decevos d'otra bona pieza, / qu'estando xecutando una ensolencia / vigo ña paré escrita so sentencia» (vss. 389 a 392).

Nesti símil endoculturador de mena regueriana, el narrador pasa de golpe a dirixise a un solu oyente. ¿Tracamundiu de la copia de la que disponemos? ¿Confusión del escritor?

«¿Xamás pasaste pel Llano del Monte / cuando cuecen el barro ña teyera? / y al llado, da u'l gorgoxo cai lla fonte, / verís abierto a modo de montera / un grandísi-

9 «iOh Baltasar, jefe de los magos!» ye la forma como na Biblia católica se dirixe Nabucodonosor a Daniel (que nesta parte del llibru ye «Baltasar»); Daniel, 4.6, en La Santa Biblia, Barcelona (Editorial Planeta), 1966.

10 "Mane, thezel, phares», diz la Vulgata Biblia Sacra iuxta Vulgatam Clementinam, Madrid (Biblioteca de Autores Cristianos), 1965, páx. 860 (Daniel, 5-25). 
mo pozo, que Aqueronte, / anque afinque so barca ña ribera / del Leteo y a Plutón mire lla cama, / ñon vería desbuchar tan grande llama» (vss. I85 a 192).

\section{Inadecuaciones / incoherencies / vulgaridaes}

Un capítulu un daqué estremáu, anque podía ser enxertáu de manera xenérica nel anterior, ye esti nel qu'atropamos inadecuaciones, incoherencies y vulgaridaes.

«Bien someya a so pá en ser devertido, [ .] / era home mui xovial y entretenido, / xugaba al tenderete y a veintiuna, / y podía, con so ardil y sin fer ruido, / pasar de balde lla ñegra llaguna, / como al son de la llira pasó Orfeo / y, polla fuerza, Alcides y Teseo» (vss. 9 a I6).

Vamos dexar de llau la impropiedá d'un narrador xefe de cuadriella (ta al frente d'una tropiella segadores) o pequeñu propietariu que fai comparances al través de la mitoloxía grecollatina. Almitamos que’l narrador nun ye'l personaxe narrador en «yo», sinón un narrador omnisciente, con capacidades enciclopédiques. Agora bien, el saltu semánticoemocional ente ser "xovial y entretenido" por xugar «al tenderete y veintiuno» y la so capacidá pa pasar «de balde» «lla ñegra llaguna» (seya lo que seya lo que signifique ehí concretamente «de balde», ensin paga-y a Caronte o ensin llucha o violencia) equival a un profundu abisu de incoherencia o desaxuste ente los dos atributos con que se lu califica, «xovial y entretenido» y capaz de «baxar a los infiernos de balde pol so ardil».

Nun tien muncha coherencia esta forma enfotadiega de pidir silenciu y atención, hasta cuatro vegaes («atendé», «¡cuenta me llamo!»-que, por cierto, nun sé mui bien qué quier dicir-, «nadie me diga chit», «icierra Candamo!»»), con espresiones, amás, que son, polo menos, un daqué incoherentes ente sí: 
«Hora bien, atendé, ¡cuenta me llamo!, / nadie me diga chit, ¡cierra Candamo!» (vss. 63 a 64 ).

Y, a propósitu, el «cierra Candamo», un gritu d'ánimu o d'exhortación a la pelea ${ }^{\mathrm{II}}$, nun paez traíu más que pola rima.

Nel segundu d'estos dos versos colos qu'entama la estrofa undécima vemos una amplificación redundante que nun paez tener otra xustificación que la construcción de la estrofa:

«Xuntánonse los magos al pescudo, / cadún con bones ganes de sabello [de desentrellizar el sueñu de Nabucodonosor]» (vss. 81 y 82).

Y nestos dos versos de la estrofa duodécima, nun hai tampoco munchu aciertu:

«Un home que apostaba [Daniel], con so treta, / del suiñu [de Nabucodonosor] desllindar tudo el lliñaxe» (vss. 93 y 94).

Llamar «treta» a la so capacidá interpretativa de los suaños, concedida, dicitur, por Dios, nun paez mui acertao o preciso. Lo mesmo podemos dicir del «lliñaxe» pa espresar el conteníu del suañu, la so sucesión d’imáxenes.

«Ñon se estevo Daniel quido un instante / ansí que lla llicencia-y fo dada, / porque ensin reparar atrás ni alante, / escopir, ñin echar lla gargaxada, / como era tan sabiudo y estodiante, / dexó a toda la xente allí ablucada / y, ensin hábitos llargos ñin bonete, / todo lo sepo como un reguilete» (vss. IOS a II2).

Equí lo más destacable ye’l nivel «vulgar» de la llingua, la inadecuación de la materia cola forma de tratala. Aceptamos que les espresiones pa les qu'adicamos («ensin reparar atrás ni alante, / escupir, ñin echar la gargaxada») puen tener una voluntá satírica o cómica, pero la so vulga-

"Ye verosímil la desplicación que da na nota 20 de la so edición Xuan Busto: «Expresión que procede del ¡Santiago y cierra!, que derivó nel ¡cierra España! de las Navas de Tolosa». 
ridá (masque esprese esa voluntá) ye mui poco afayadiza cola «seriedá» de la materia tratada. Del mesmu mou, el «ensin hábitos llargos ñin bonete» (ensin tener estudios clericales, como anota Xuan Busto) ye tamién una espresión d'un nivel escesivamente «d'andar per casa».

Atopamos na estrofa I9 esta forma de precisar que Misael, Ananíes y Azaríes salieren indemnes del fornu nel que los echaren por orde de Nabucodonosor:

«A tres mocicos qu'illi conociera, / Misael, Ananíes y Azaríes, / llos que en forno baillano(n) lles folíes» (vss. I50 a I52).

Lo que dicen les versiones católiques ${ }^{12}$ del Llibru de Daniel ye que cantaron "Cánticos al Señor». El canciu ye un testu d'emponderanza de Dios pola so obra y d'invitación a lloalu y aponderalu por ello. D’ehí, evidentemente, a «baillar lles folies» hai una bona ralura, y esi «baillar lles folíes» da-y un tonu cómicu, sinón vulgar, a esi momentu del testu.

Tamién los versos primeru y segundu de la estrofa 22, «Ñon-y causó bona prestia al rei el cuento / porque arañaba el sayo, como un gato», constituyen un exemplu d'estilu que nun se compadez cola seriedá del tema nin tienen correspondencia col testu bíblicu, que diz que «Entonces Nabucodonosor se llenó de ira y, demudada la expresión de su rostro contra Shadrak, Meshakh y Abed-Negó [Misael, Azaríes, Ananíes], tomó la palabra y mandó encender el horno siete veces más de lo que se acostumbra a encenderlo» ${ }^{13}$.

En «Lluigo que fue escubierta lla maraña» (estrofa 23, primer versu) «lla maraña» ye una espresión escasamente afayadiza, mui impropia, so Biblia.

${ }^{12}$ Delles versiones protestantes tienen estos cánticos como apócrifos y nun los enxerten na

${ }^{13}$ Daniel, 3.19, en La Santa Biblia, Barcelona (Editorial Planeta), I966. 
pa designar la negativa de los trés mozos a adorar la estatua d'oru que Nabucodonosor llevantara.

Un nivel percoloquial ye'l de "cual Pedro per so casa» en

«Pasiaben el camín, qu'eso diz qu'era, / como dicen, cual Pedro per so casa» (versos primeru y segundu de la estrofa 26).

Y poco axeitáu ye’l sintagma "pasiaben el camín» pa dicir qu’andaben dientro del fornu.

El coloquialismu más vulgar apaez otra vegada nos versos que vienen agora, col símil de "cangrexos so la brasa». Tampoco ye mui afortunáu el «cantanon ensin tasa»:

«cantaben y baillaben de manera / que parecín cangrexos so la brasa, / solo que nestos ye la voz postrera, / pero aquellos cantanon, ensin tasa» (versos terceru y cuartu de la estrofa 26).

A partir de la estrofa 3I, entama la narración pol rei Nabucodonosor -en primer persona, poro, como nel Llibru de Daniel- d'un suañu, un segundu suañu. Y ye nesti tramu del poema onde s'atropen delles fórmules espresives, dellos elementos de cita cultural y dalgún de mena comunicativa que resulten dafechamente estrañes o poco afayadices. Por exemplu, les cites pol rei de les histories mitolóxiques del mundu clásicu de Bato, Admeto y Mercurio; la calterización del camaleón [estrofa 35] coles traces fabuloses coles que más tarde se lu va a pintar; la mención perifrástica d'Eos ${ }^{14} \mathrm{y}$ les de Titón y Laomedonte. Too ello, nun fai falta dicilo, nun ta nel testu bíblicu.

Ye discutible tamién, dende'l puntu de vista de l'adecuación del discursu tanto al testu bíblicu como a la verosimilitú del propiu discursu, el

\footnotetext{
${ }^{14}$ Asina ye: «Mas muéva-yos tamién el desperdicio / de la lloramicona, que del viento / diz que ye madre, y de Titón fenoya» (vss. 293 a 295).
} 
que Nabucodonosor s'esprese -al marxe de les cites mitolóxiques- como un contemporaneu (rural) de Balvidares:

"Yo vi un árbol tamaño y tan pompioso / que'l carbayo mayor con illi é faba» (vss. 24I-242).

«que panera ñon hai tan atacada» (vs. 246).

"que fese podía / a so solombra cualquiera romería» (v. 248).

"o esti un santo [venía] dando voces, que podía / oíse dende Uviedo hasta Llugones» (vss. 283-284).

Y tampoco falten espresiones un daqué xostrones o imprecises, como la de

"porque nilli [nel árbol] encontraben, sin fatiga, / cuanto-yos abarcaba lla barriga» (vss. 27I y 272).

O esti adínatu o impossibilis:

"[convivíen] cabres, xatos, oveyes y ñuvielles, / con llos osos y llobos per ente elles» (vss. 255 y 256 ).

Les espresiones de coloquialismu vulgar o d'inadecuación de lo dicho colos personaxes continúen per ayures. Asina, na estrofa 39, esti:

«Aquesto me pasó, Daniel amigo, / ñon quiero que te duermes eñes payes, / rumia bien el pasaxe allá contigo» (vss. 305 a 308).

"Que mios sabios, por más qu’echen el resto, / ¡diablo!, palabra acierten de todo esto» (vss. 3II y 3I2).

Onde, además de lo poco apropiao del llinguaxe pal rei, queda esi increíble «Daniel, amigo», na apelación real. Tutéu, que por cierto, repite Daniel na estrofa 46 al dirixise al rei asina:

«—Pollo cual, rey, amigo, ñon te embobes—, / -y dixo—, ñi a mal tengues mio conseyo".

$\mathrm{Na}$ estrofa 40 Daniel, dándo-y vueltes al suañu de Nabucodonosor, 
"casi una hora se vio sin topar brecha / esmesándose les uñes con llos dientes» (vss. 315 y 316$)$.

(Anque tien gracia esa creación metafórica d'«esmesar les uñes colos dientes»).

Esti [estrofa 4I] «peruyada» metafóricu pa espresar les ganancies y triunfos que diben llevar los enemigos del rei:

"y que a sos enemigos, en concencia, / mui bona peruyada-yos cayera» (vss. 325 у 326$)$

O esti otru coloquialismu (tan «modernu», d'otru llau, pol conceptu y la palabra d'acidiente, 'muerte repentina'):

«Porque se [lléase si] te lo cuento de repente / pienso que t'has quedar nun acidiente» (vss. 343 y 344 ).

La parte final del parlamentu de Daniel nel qu'interpreta'l segundu suañu de Nabucodonosor, afátase, como la mayor parte de la «versión» del testu bíblicu, con elementos d'endoculturación; pero, pese a que podemos entender eso, nun paez que les imáxenes y oxetos (y palabres que les designen, polo tanto) escoyíos pa ello correspuendan col nivel del discursu esperáu pa un testu d'esti calter. Estos son los conseyos [estrofa 46]:

"Apurre bonos gaxos a los probes / y tápa-yos con sayos el pelleyo, / que si ansí lo xecutes no habrá escobes / en toda Babilonia y so conceyo / que barrien un desván, cuarto o bodega, /como to veluntá lliberaliega» (vss. 363 a 368).

Nel Píramu y Tisbe que conocemos malapenes esisten estos defectos (vid. infra). Sí tienen en común delles coses, toes elles biltos de Marirreguera: l'entamu endoculturador onde nun llugar indetermináu asturianu, rural, un emisor se dirixe a los sos oyentes pa conta-yos un "cuentu» 
o historia; l'emplegu de símiles de raigañu homérico-virxilianu ${ }^{15}$, tamién usual en Marirreguera, el cierre de la obra con una nueva parénesis a los oyentes-circunstantes. Si acaso, el Píramu y Tisbe presenta una mayor complexidá estructural, porque al final del relatu, que pieslla cola apelación a los oyentes-circunstantes yá dicha, añádese una nueva octava, onde apaez un narrador esternu pa facer una valoración de la vida rural, dientro'l recurrente tópicu lliterariu del aponderamientu de l'aldea.

Verdá ye que Los sueños... cierren tamién con unos versos estraños a la materia narrada, pero nesti casu nun se trata d'un discursu relativu al propiu poema, internu, sinón una excusatio o captatio benevolentiae, pidiendo disculpes (y, amás, en castellanu) polos erros o faltes del testu.

Brevemente, vamos desaminar dalgunos otros de los aspectos coincidentes ente los dos testos (Píramu... y Sueños...), asina como la so semeyanza colos demás testos de Reguera. Tocántenes al narrador internu, al protagonista narrador, llama equí l'atención el que tenga nome (Xuan García) y la so calterización social y cultural («que yera amu de casa y home honradu (sabía lleer, escribir tamién sabía, / y aún daqué de llatín tenía estudiadu)»), lo que nun sucede en dala de les obres nes que tamos peñerando. Nesi sen, nun ofrez esi datu razones p'averar Píramu a un autor o a otru. En cuanto a l'apelación a los oyentes circunstantes con nomes propios en Píramu... y Sueños..., tampoco podemos sacar conclusiones. Si acaso, apuntar qu'en Sueños... la combinación de personaxes apelaos y acciones que se-yos piden o coses que se-yos refierten o esixen ta más espardida. Tanto Píramu... como Sueños... tienen al final del poema una nueva parénesis personificada a los oyentes-circunstantes, a ún nel casu los Sueños... («Atira acá, Mingón, lla tabaquera», v. 385), a cuatro en Píramu... (Antón, Xuanín, Pachu, Maruxa, vss. 229 a 235).

\footnotetext{
is Esti, nos versos 89 a Iоo: “iNon visteis de la tierra pel veranu / desque cayen algunes goteriques..."
} 
Nin Dido y Enees nin Hero y Lleandro, que sí tienen narrador endoculturador con apelación a los oyentes, nun tienen una conclusión con parénesis a oyentes-personaxes específicos, anque sí de forma xenérica.

Per otru llau, tienen un paecíu bultable estes apelaciones del entamu de Dido y Enees («Ola, Tuxa, ¡tu riste? Pos bien oyes; / saca dos filos, toma aquella rueca; / Xuanín, enriestra allí cuatro panoyes; / Bartuelu, tapa a Ruda aquella llueca») coles de Píramu nesa mesma circunstancia: «—XXuanín!, cierra esa esa puerta, que fai vientu. / ¡Llarina!, ve unes breces encendiendu. / Mete un felechu, Roque, ña cencerra / al xatu, y arrecueye aquesa perra». ¿Zunes escrituraries del mesmu autor? ¿Influencia del autor más antiguu sobre'l modernu?

L’usu de la mitoloxía ye estremáu. Frente al «abusu» de los Sueños..., como viemos enriba, l'autor de Píramu nun emplega más qu'una cita de la historia de Vertumno y Pomona ( $\mathrm{E}$ l frutu con que a simples acallenta / de Vertumno lo ichó de ver Pomona, / que dempués que ñon pudo como vieyu, / llogró remocicándose el pelleyu»), nomes que, por cierto, evoca tamién l'autor de los sueños («Folgábase Ñabuco a so placencia, / como solía Vertumno con Pomona, (vss. 227 y 228)»), colo que podemos facenos la mesma entruga: ¿vezu d'un mesmu autor o influencia? Anque ye verdá que l'emplegu lliterariu de la mitoloxía clásica ye un recursu universal na época.

(Per otru llau, tamién Hero y Lleandro emplega la mitoloxía, y de forma nun escasa, nesti pasaxe concretu que vien agora, como elementu de referencia pa comparar a Hero con otres muyeres de la mitoloxía clásica, nun pasaxe, por cierto, escurucu en dalgunos versos: «¿Qué tuvo que ver la otra mentecata, / que dicen qu'era fresca como un oro, / si codició volvese en una xata / para poder folgase con un toro? /; ¿nin la otra, tamién más insensata, / que s'echó so la espada sin decoro / de pedir para fello lla llicencia, / ñon por Píramo, Tisbe, nin Llucrencia? / ¿Nin la otra que so padre lla guardaba / eña torre de fierro mui severo, 
/ que en granicos de sol la soliviaba / Xúpiter, qu'era Dios mui braguetero? / ¿Nin la otra que los homes amudaba / cual en llobo cerval, cual en carnero, / con ésta que yá sube a los balcones / a ver cómo lo fan los farfantones?» vss. II3 a I28 ${ }^{16}$.

L'elementu más distintivu ente un testu y otru son, ensin dulda, lo que llamamos nel Sueños... «imprecisiones, inadecuaciones, incoherencies y vulgaridaes», que malapenes esisten nel Píramu y Tisbe. Yo nun soi pa señalar nesti más que dos: la cita mitolóxica de Vertumno y Pomona («El frutu con que a simples acallenta / de Vertumno lo ichó de ver Pomona, / que dempués que ñon pudo como vieyu, / llogró remocicándose el pelleyu»), onde'l que nun conoza la historia desconoz qué quier dicir el testu, y entá conociéndola, esiste una elipsis tan bultable que la espresión ye bien imprecisa ${ }^{17} ; \mathrm{y}$, acaso, esi «fumo como de cuchu de moñiques» del versu 92, anque tengo les mios duldes de qu'equí, al calificalo asina, nun tea yo aplicando una visión hodierna que nun co-

${ }^{16}$ La comparanza faise n'ocasiones cola belleza de los referentes, pero, más bien, la mayoría les veces paez facelo cola so conducta, cola so «normalidá». Por cierto, l'escuru versu I2o provocó delles llectures nos manuscritos de que disponemos: «ñon por Píramo, Tisbe, nin Llucrencia?» (CA)»; «Nin Pírame, nin Thisbe, nin Llucrencia?» (CD), «ñin la Príamo Tisbe, ñin Llucrencia?» (CE); «ñin la Príamo Tisbe, ñin Llucrencia?» (BC); y Caveda (al mio xuiciu, la peor llectura): «nin Píramo, nin Tisbe, nin Llucrencia?». Lo que faen los versos iı7 (¿«ñin la otra, tamién más insensata...?») al I20, el que tamos viendo, ye la comparanza con muyeres famoses y guapes que se suicidaron (la comparanza, por cierto, vien ser anticipadamente irónica, porque Hero va suicidase tamién tirándose al vaciu) dándose muerte pola propia mano con un fierru: Dido, Llucrencia y Tisbe. Dende esi puntu de vista, el testu que más s'aproxima parcialmente a una escritura recta ye’l de BC: "ñin la Príamo Tisbe, nin Llucrencia», si bien ye necesario restituir ehí "Príamo» por "Píramo», onde lleeríamos entós «la Tisbe de Píramo», n’hipérbaton. Si se me permite vo facer, entós, un par de propuestes que faen más clara la llectura: «de pedir pa fello lla llicencia (Dido), / (ñin de Píramo, Tisbe), nin Llucrencia?», onde s'arreya'l sintagma «ñin de Píramo, Tisbe» con "pedir la llicencia» (pa espetase’l fierru); y «ñin de Píramo Tisbe, nin Llucrencia», na que Tisbe y Llucrencia son una más de la llista de suicides pol fierru, solo qu’a Tisbe calterízasela como «Tisbe de Píramo», n’hipérbaton.

${ }_{17}$ Masque, nun se nos escaeza, toes eses hestories son "pastu común» de la xente ilustrao de la época (pero non de los campesinos, a los que según dalgunos, pretendíase adoctrinar con estes histories). 
rrespuende cola época, sobre too, si tenemos en cuenta que, una vegada escoyíu l'elementu la realidá sobre’l que se llevanta'l símil, nun paez haber otra manera de dicilo.

Paez, en consecuencia, perdifícil que la persona qu'escribe los Sueños de Nabucodonosor seya la mesma que la qu'escribe Piramu y Tisbe, incluso nel casu que quedramos suponer nella una evolución mui positiva a lo llargo los años. Lo que vien llevanos a esa impresión que, según manifesté al principiu d'esti testu, fue la que tuvimos Antón García y yo al lleer dambos los Sueños... la vegada primera, y la qu'asoleya tamién Pablo Suárez García al través del análisis estadísticu.

\section{LOS ARGUMENTOS}

Tanto Caveda (I839) como Canella (I887) alluguen Píramu y Tisbe nel cuellu de Marirreguera. Ye Xulio Viejo Fernández (1997) el primeru que nun lo fai, escluyéndolu na so edición de la obra completa del carreñense $^{\mathrm{r} 8}$. Les sos razones, que vamos desaminar darréu, póngoles en cuestión en 2004, nel mio Les producciones clasicistes d'Antón de Marirreguera $^{19}$. Con datos nuevos, Xuan Busto asoleya'l Piramu... en 2012 ente la obra conocío de Benito de l'Auxa ${ }^{20}$.

L’argumentación de Xulio Viejo ye, en sustancia, la siguiente: González Posada atribuye la obra a Marirreguera na so Biblioteca Asturiana y nes sos Memorias históricas (si bien equí failo siguiendo'l testimoniu de Fernández-Porléi, y diciendo que la tala fábula -de Píramu y Tisbe- «yá

${ }^{18}$ Antón de Marirreguera. Fábules, teatru, romances (edición, introducción y notes de Xulio Viejo Fernández), Uviéu (Alvízoras Llibros), 1997. Los sos argumentos, ente les páxines 36 a 40.

I9 Xuan Xosé Sánchez Vicente, Les producciones clasicistes d'Antón de Marirreguera, Trabe Ediciones, Uviéu, 2004. Les mios razones, de la páxina II6 a la I3I.

20 Benito de l'Auxa, Antón Balvidares. Poesies (edición, introducción y notes de Xuan Carlos Busto, Uviéu (Ediciones Trabe), 2012. 
no se encuentra», esto ye, copies d'ella). Agora bien, na mesma Biblioteca asturiana fala d'un Píramu y Tisbe d'Auxa qu'entama por «era de octubre la noche primera», que Viejo entiende como una castellanización del «La postrer nuichi yá d'octubre yera» del testu que güei conocemos, que sería, polo tanto, el d'Auxa ${ }^{21}$.

Amás convién, enantes de siguir, precisar dalguna cuestión. La primera, que Xovellanos, amigu d'Auxa y al qu'esti-y dedica los Sueños..., da’l Píramu... como de Reguera. La segunda, que Caveda, que remanó seguramente tolos manuscritos de la época -los más comunes y los más raros, los más estropiaos y los meyor conservaos, los qu’asoleyó na so esbilla de i839 y los que desdexó- manifiesta que «el señor Posada ya citado dice que ya no se encuentra esa poesía: en el día es tan común como las otras; yo tengo un ejemplar y he visto algunos otros» ${ }^{22}$. Otra más, que yo recueyo nes páxines II7 y iI8 de la mio obra marirregueriana, ye la esistencia d'una publicación fecha por Enrique Gil y Carrasco nel Semanario Pintoresco Español. Son una mozadina versos que tienen la intención d'amosar al públicu de la revista lo que ye una esfoyaza. Esos versos conociólos él en Cangues d'Onís (hai que suponer que vería'l poema enteru), l'añu anterior a l'asoleyamientu nel Semanario... y a la del recopilatoriu de Caveda y Nava, en $1838^{23}$. Pues bien, esos versos qu'entamen pol "era d'octubre la noche postrera»

${ }^{21}$ Por cierto, Xulio Viejo trescribe'l so nome como Benito de la Uxa; Xuan Busto, Benito de l'Auxa.

${ }^{22}$ La nota tómase de la páxina 38 del llibru de Xulio Viejo. El testu pertenez a un estudiu previu de Caveda, en I824, a lo que fue l'entamu a la so esbilla de I839.

${ }^{23}$ L'artículu ta fecháu'l 8 de payares de I818 en Cangues. Tomo la nota d'Asturias vista por viajeros románticos extranjeros y otros visitantes y cronistas famosos. Siglos $X V$ al XX (introducción, selección y notas de José Antonio Mases, Gijón (Ediciones Trea), 20or. L'artículu de Gil y Carrasco, nes páxines 279 a 286 del primer tomu; los versos, na 282. 
nun son los mesmos que los qu'asoleya Caveda nin los qu'apaecen en nengún de los manuscritos conocíos hasta agora, lo que quier dicir que los manuscritos de Píramu... corríen n’abondanza, escontra lo que diz Posada ${ }^{24}$.

Xulio Viejo arreya un par de razones más, de non munchu pesu. El primeru, que Canella, na so Historia de la Universidad de Oviedo, de I873, nun cita Píramu y Tisbe ente les poesíes conocíes del candasu; ensin embargu, cuatro años más tarde reedita l'antoloxía cavediana, con dalgunos ensiertos y delles notes, ensin correxir l'autoría de Reguera. El segundu, que'l manuscritu llamáu CA por Viejo (qu’anduvo nes manes de Caveda) y que recueye obres de González Reguera, nun atropa nin el Pleitu ente Uviéu y Mérida nin Píramu y Tisbe. Evidentemente, que nun atrope Píramu y Tisbe nun ye razón dala pa suponer que nun ye del pá la nuestra lliteratura, porque, si lo fuere, ¿qué conclusión podíamos sacar de que nun acueya'l Pleitu..., la primer piedra l'edificiu la lliteratura asturiana?

El restu de los razonamientos, d'análisis internu y cadarma'l testu, que, me paez, quedaben refugaos o, polo menos, mui puestos en dulda, nel mio esame de 2004, talanto que queden perrefugaos, o más puestos en dulda, col análisis del testu los Sueños de Nabucodonosor qu'enriba ficimos. Y lo mesmo podía dicise de los que, dende esos puntos de vista, fai Xuan Carlos Busto na so edición de los poemes de Benito de l'Auxa y de Balvidares.

Por esponelo too, hai que dicir que Xulio Viejo entrúgase si'l Pira$m u .$. de que disponemos ye enteru de Benito de l'Auxa o esti lo que fixo fue trabayar sobre'l de Reguera y modificalu.

${ }^{24}$ Hai que pensar, además, que Posada escribe la mayor parte la so obra dende fuera d'Asturies, colo que pue que, en dacuando, los sos datos sean de memoria o d'oyíes, y, polo tanto, imprecisos. 
Con too, l'argumentu más fuerte a favor d'Auxa son los dos manuscritos de que Xuan Busto, el CF y CD, nos da cuenta qu'atribuyen el Píramu y Tisbe a Auxa. Asina nos diz, por exemplu, el que'l críticu llama CF: «Fábula de Píramo, y Tisbe, en Dialecto Asturiano [...] Égloga en dialecto bable asturiano, por don Benito de la Auja Manuel Presvo . y natural de Candas, en Carreño».

\section{Conclusiones}

Pese a los dos manuscritos que tienen por autor de Piramu y Tisbe a Benito de l'Auxa y a les contradictories declaraciones de Posada, paez difícil caltener l'autoría del coetaneu de Xovellanos, ante too poles diferencies de capacidá y estilu qu'esisten ente los Sueños... y el Píramu..., según viemos enriba. Ye verdá que podíamos suponer que los Sueños... son obra d'un autor inmaduru, que diría evolucionando hasta facese un escritor d'otra mena na so madurez. Ye posible, pero difícil.

L'otru puntu decisivu ye la posición Caveda no respective a l'autoría. Pa entamar, vamos recordar qu'a les palabres de Posada diciendo que yá nun había manuscritos ("yá no se encuentra») del Píramu... de Reguera, el villaviciosín respuende directamente, como enriba trescribimos, que «el señor Posada ya citado dice que ya no se encuentra esa poesía: en el día es tan común como las otras; yo tengo un ejemplar y he visto algunos otros». Talántese bien lo que diz: que sí hai copies del Píramu... del entamador de la nuestra lliteratura, de Marirreguera, y qu'él tien una.

Puntu difícil de desplicar ye por qué Caveda, que tuvo, según sabemos, nes sos manes los manuscritos CF y CD, onde l'autoría dáse-y de forma esplícita al coetaneu de Xovellanos y de so padre, Caveda y Solares, refuga esta atribución. Podía pensase en dalgunes razones. Una d'elles, la de que considerare qu'allugando l'autoría en Reguera, la figura d'esti salía reforzada y que los trés testos (Hero y Lleandro, Dido y Enees, Píramu y Tisbe) embaxo la firma d'un mesmu autor teníen mayor 
coherencia pa la presentación en sociedá de la lliteratura asturiana. Pero d'otru llau, podía ser tamién que, conociendo los Sueños..., pensare, como nós, qu'un autor con tantes deficiencies d'escritura nun podía ser el mesmu del Píramu y Tisbe qu'acabó asoleyando (al mesmu tiempu, refugaríalu de la nómina de la esbilla y nun asoleyaría los Sueños..., lo mesmo que rechazó La Xudí de González Villar pa reescribir él otra).

Pero tal vez paez más cenciello entender que Caveda y Nava, al marxe del so taste lliterariu, tenía fontes d'información bayuroses, tanto testuales como tradicionales, al través de so padre, de los amigos de so padre y de les noticies de la xente del Institutu puestu en marcha por Xovellanos, pa tar seguru de que Píramu y Tisbe, pese a los datos contradictorios, yera obra de Reguera.

Inclusive, anque supiere que'l Píramu y Tisbe que conocemos, el qu'él asoleya y que ye prácticamente igual en tolos manuscritos, tuviese, na so forma conocida, dalgún arreyu o retoque del escritor del xviII. 

